\title{
Involvement of NLRP3 inflammasome in the impacts of sodium and potassium on insulin resistance in normotensive Asians
}

\author{
Zhaofei Wan†, Wen Wen†, Keyu Ren†, Dong Zhou, Junhui Liu, Yue Wu, Juan Zhou, Jianjun Mu* and \\ Zuyi Yuan* \\ Department of Cardiovascular Medicine, First Affiliated Hospital of Medical College, Xi'an Jiaotong University, Xi'an, Shaanxi, \\ 710061, People's Republic of China
}

(Submitted 6 December 2016 - Final revision received 14 September 2017 - Accepted 26 September 2017)

\section{Abstract}

Salt, promoting oxidative stress, contributes to insulin resistance, whereas K, inhibiting oxidative stress, improves insulin sensitivity. Oxidative stress activation of NLRP3 inflammasome is a central player in the induction of insulin resistance. Therefore, we hypothesised that NLRP3 inflammasome may mediate the effects of salt and $\mathrm{K}$ on insulin resistance. In all, fifty normotensive subjects were recruited from a rural community of Northern China. The protocol included a low-salt diet for $7 \mathrm{~d}$, then a high-salt diet for $7 \mathrm{~d}$ and a high-salt diet with $\mathrm{K}$ supplementation for another $7 \mathrm{~d}$. In addition, THP-1 cells were cultured in different levels of Na with and without K. The results showed that salt loading elevated fasting blood glucose, insulin and C-peptide levels, as well as insulin resistance, whereas K supplementation reversed them. Meanwhile, additional K reversed the active effects of high salt on NLRP3 inflammasome in both the subjects and THP- 1 cells, and the change of insulin resistance index notably related with the alteration of plasma IL- $1 \beta$, the index of NLRP 3 inflammasome activation, during intervention in the subjects. Additional K ameliorated oxidative stress induced by high salt in both the subjects and cultured THP-1 cells, and the change of oxidative stress related with the alteration of plasma IL- $1 \beta$ during intervention in the subjects. In vitro, antioxidant $\mathrm{N}$-acetyl-L-cysteine significantly prevented the active effects of high Na or oxidant Rosup on NLRP3 inflammasome, so did K. Our study indicates that oxidative stress modulation of NLRP3 inflammasome may be involved in the impacts of Na and $\mathrm{K}$ on insulin resistance.

\section{Key words: Insulin resistance: NLRP3 inflammasome: Oxidative stress: Sodium: Potassium}

It is universally accepted that populations with high-salt ${ }^{(1-4)}$ and indigent- $\mathrm{K}^{(5-7)}$ diet are more predisposed to developing CVD. In addition, it is well known that insulin resistance has been recognised as a cornerstone of CVD occurrence and development ${ }^{(8,9)}$. In fact, excess salt intake contributes to insulin resistance ${ }^{(10-14)}$ and $\mathrm{K}$ supplementation exerts a protective effect against insulin resistance ${ }^{(15-17)}$. However, the mechanism underlying the completely inverse impacts of $\mathrm{Na}$ and $\mathrm{K}$ on insulin resistance remains poorly understood.

IL-1 $\beta$ not only interferes with the signalling of insulin target tissues such as liver, muscle and adipose tissues ${ }^{(18-20)}$, but also directly promotes pancreas islet beta-cell dysfunction and cell death $^{(21)}$. Therefore, NLRP3 inflammasome, serving as the key platform for IL-1 $\beta$ maturation and secretion, plays a central role in the initiation and progress of insulin resistance and diabetes ${ }^{(22,23)}$.

Various danger signals and environmental irritants activate NLRP3 inflammasome through triggering reactive oxygen species (ROS) generation ${ }^{(24-26)}$. It is worth noting that an increased oxidative stress occurs in rats with high-salt diet ${ }^{(27-30)}$, whereas high-K diets decrease oxidative stress in rats ${ }^{(31)}$ and $\mathrm{K}$ can inhibit ROS generation of human white blood cells in vitro ${ }^{(32)}$. Therefore, we hypothesised that Na could activate NLRP3 inflammasome by enhancing oxidative stress and further result in insulin resistance, whereas $\mathrm{K}$ could moderate the effect. In the present study, fifty normotensive subjects were recruited and received an intervention trial with three phases, including low-salt diet for $7 \mathrm{~d}$, high-salt diet for $7 \mathrm{~d}$ and high-salt diet with $\mathrm{K}$ supplementation for another $7 \mathrm{~d}$ in normotensive subjects, and THP cells were cultured in different levels of $\mathrm{Na}$ and $\mathrm{K}$ to determine whether NLRP3 inflammasome is involved in the impacts of $\mathrm{Na}$ and $\mathrm{K}$ on insulin resistance.

\section{Methods}

\section{Subjects}

We enrolled fifty subjects, aged 29 to 65 years, from a rural community of northern China. One of them was excluded because the subject failed to finish whole intervention procedures and so the data of forty-nine subjects were analysed finally. Main demographic data, such as age, sex, weight and waist circumference, were collected. The criterion for hypertension was a resting blood

Abbreviations: BP, blood pressure; MDA, malondialdehyde; ROS, reactive oxygen species; SOD, superoxide dismutase; SS, salt sensitive; SR, salt resistant.

* Corresponding authors: Z. Yuan, email zuyiyuan@mail.xjtu.edu.cn; J. Mu, email mujjun@163.com

$\dagger$ These authors contributed equally. 
pressure (BP) of over 140/90 $\mathrm{mmHg}$ at two different visits or on medication. Exclusion criteria were as follows: advanced liver disease, renal failure, cancer, valvular heart disease, heart failure, stroke, atrial fibrillation, peripheral arterial disease and other vascular diseases. The investigation conformed to the Declaration of Helsinki. The study protocol was approved by the Institutional Ethics Committee of Xi'an Jiaotong University Medical School and written informed consent was obtained from all the subjects.

\section{The protocol of population intervention}

As described previously ${ }^{(7,33,34)}$, the protocol included a questionnaire survey and physical examination for $3 \mathrm{~d}, 7 \mathrm{~d}$ on a low-salt diet $(3 \mathrm{~g}$ of $\mathrm{NaCl} / \mathrm{d}), 7 \mathrm{~d}$ on a high-salt diet $(18 \mathrm{~g}$ of $\mathrm{NaCl} / \mathrm{d}$ ) and a high-salt diet with $\mathrm{K}$ supplementation $(4.5 \mathrm{~g}$ of $\mathrm{KCl} / \mathrm{d}$ ) for another $7 \mathrm{~d}$. Before the investigation, all subjects were given detailed instructions to avoid cooking salt, table salt and salty snacks during the entire intervention period. All of the meals were cooked in research kitchens and consumed onsite under the supervision of the study staff.

\section{Blood pressure measurement and definition of salt sensitivity}

Three BP measurements were performed every morning with a Hawksley random-zero sphygmomanometer during the 3-d baseline observation period and on days 2, 5, 6 and 7 of each intervention period. BP was measured by trained and certified members according to a standard procedure recommended by the American Heart Association. BP was measured after $5 \mathrm{~min}$ of rest. In addition, all subjects were warned to avoid cigarette, alcohol, coffee/tea and exercise for at least $30 \mathrm{~min}$ before BP measurement. Salt sensitivity was defined as a mean arterial BP (MAP) increase of more than $5 \mathrm{mmHg}$ from a low- to a high-salt diet intervention ${ }^{(33,35)}$.

\section{ELISA}

Fasting venous blood samples were drawn by trained phlebotomists in the morning of day 7 of each intervention period and were collected in EDTA-vacutainer tubes. The plasma samples were centrifuged at $3000 \mathrm{rpm}$ for $20 \mathrm{~min}$ at $4^{\circ} \mathrm{C}$ and were then kept frozen at $-80^{\circ} \mathrm{C}$ until analysis. The supernatants were collected and stored at $-80^{\circ} \mathrm{C}$. Plasma and the supernatant level of IL- $1 \beta$ was detected by ELISA with Human IL- $1 \beta$ ELISA Kit (Biolegend and R\&D, respectively).

\section{Assessment of insulin resistance}

Plasma insulin and plasma C-peptide were detected by radioimmunoassay $\left({ }^{125} \mathrm{I}\right.$ Insulin Radioimmunoassay Kit or ${ }^{125} \mathrm{I}$ C-peptide Radioimmunoassay Kit; Beijing North Institute of Biological Technology). Plasma glucose was assayed by our hospital's clinical laboratory. Homeostatic model assessment for insulin resistance index (HOMA-IR) was calculated using HOMA Calculator version 2.2.3 software.

\section{Oxidative stress assay}

Plasma superoxide dismutase (SOD; Nanjing JianCheng Bioengineering Institute) and malondialdehyde (MDA, Nanjing JianCheng Bioengineering Institute) were determined by colorimetry.

\section{Isolation of peripheral blood mononuclear cells}

Peripheral blood mononuclear cells (PBMC) were isolated by Ficoll standard density gradient centrifugation. The upper layer containing PBMC was harvested and washed with Hank's balanced salt solution and then with PBS.

\section{Cell culture}

THP-1 cells were cultured in Roswell Park Memorial Institute (RPMI) 1640 medium supplemented with $10 \%$ FCS, $2 \mathrm{~mm}$ L-glutamine, 100 units $/ \mathrm{ml}$ penicillin, $100 \mu \mathrm{g} / \mathrm{ml}$ streptomycin and $50 \mu \mathrm{m} 2$-mercaptoethanol. Before intervention, cells were cultured in serum-free 1640 medium for $24 \mathrm{~h}$, so that the cell were synchronised. Pre-treated for $3 \mathrm{~h}$ with lipopolysaccharide (LPS, $200 \mathrm{ng} / \mathrm{ml}$; Sigma), THP-1 cells were then cultured with different concentrations of sodium chloride with or without potassium chloride. To assess the role of oxidative stress in the activation of NLRP3 inflammasome, THP-1 cells were pre-treated for $20 \mathrm{~min}$ with antioxidant $\mathrm{N}$-acetyl-Lcysteine (NAC, $25 \mathrm{~mm}$; Sigma) and then cultured with sodium chloride. In addition, to evaluate the role of oxidative stress in the inhibition of NLRP3 inflammasome by potassium chloride, THP-1 cells were pre-treated for $24 \mathrm{~h}$ with potassium chloride $(60 \mathrm{~mm})$ and then cultured with oxidant Rosup $(50 \mu \mathrm{g} / \mathrm{ml}$; Fanbo Biochemicals). The supernatants were collected for determination of IL- $1 \beta$. Cell viability after treatment with indicated agents was evaluated by trypan blue exclusion and was consistently $>90 \%$. All experiments were repeated three times.

\section{Quantitative PCR and Western blot analysis}

Total RNA was isolated by use of Trizol reagent (Invitrogen) and proteins were isolated by use of RIPA Cell Lysis Reagent (GENSHARE LOGICAL). The extracted RNA was reverse transcribed and analysed by quantitative PCR with use of the SYBR ${ }^{\circledR}$ PrimeScript TMRT-PCR Kit (TaKaRa). Glyceraldehyde-3-phosphate dehydrogenase (GAPDH) was a housekeeping gene. The primers used in quantitative PCR are as follows: NLRP3-F: 5'-CTGTAA CATTCGGAGATTGTGGTT-3'; NLRP3-R: 5'-GACCAAGGAGAT GT-CGAAGCA-3'.CASEPASE-1-F:5'-CCGCAAGGTTCGATTTTCA-3'; CASEPASE-1-R:5'-ACTCTTTCAGTGGTGGGCATCT-3'.GAPDH-F: 5'-GCACCGTCAAGGCTG AGAAC-3'; GAPDH-R:5'-TGGTGAA GACGCCAGTGGA-3'. For Western blot analysis, protein extracts were separated by SDS-PAGE and the transferred blots were incubated overnight at $4^{\circ} \mathrm{C}$ with caspase- 1 antibody (Cell Signaling) or NLRP3 antibody (Cell Signaling), and then horseradishperoxidase-conjugated anti-goat and anti-rat, or anti-rabbit secondary antibodies. The enhanced chemiluminescent substrate was used to detect protein bands.

\section{Reactive oxygen species detection}

The intracellular ROS was detected by use of Reactive Oxygen Species Assay Kit from Fanbo Biochemicals. The cell pellets were resuspended in $600 \mu \mathrm{l}$ of ROS detection solution for incubaton for $20 \mathrm{~min}$ in the dark, and then determined by flow cytometry (FACSanto ${ }^{\mathrm{TM}} \mathrm{II}$; BD Biosciences) with excitation 
at $490 \mathrm{~nm}$ and emission at $525 \mathrm{~nm}$. The data were obtained and analysed with the BD FACSDiva software (BD Biosciences).

\section{Statistical analyses}

Data were collected and analysed with SPSS 13.0 for Windows (SPSS Inc.). Quantitative variables were expressed as mean values and standard deviations. Categorical variables were expressed as frequency and percentage. The Kolmogorov-Smirnov test was used to assess normal distribution of quantitative variables. Independent-samples or paired $t$ tests were performed to compare the parametric values between the two variables, and categorical variables were compared by the $\chi^{2}$ test. Repeatedmeasures ANOVA was used for comparison of multiple variables. The degree of association between two continuous variables was analysed using Pearson's correlation coefficients. All probability values were two-tailed. A value of $P<0.05$ was considered statistically significant.

Results

\section{Profiles of studied subjects}

In all, twenty-one of forty-nine subjects, experiencing an increase in MAP by more than $5 \mathrm{mmHg}$ from a low- to a highsalt diet intervention, were defined as SS and the other twentyeight, experiencing less change, were classified as $\mathrm{SR}^{(33)}$. Baseline systolic BP in SS subjects was significantly higher than that in SR (Table 1,P=0.044), and compared with SR subjects the SS subjects were older (Table $1, P=0 \cdot 005$ ). There was no significant difference between SS and SR subjects in BMI, diastolic, MAP and other biochemical markers (Table 1).

\section{Potassium supplementation alleviated insulin resistance} caused by salt loading in the subjects

Compared with low-salt diet, the levels of plasma glucose, insulin and C-peptide were significantly increased after high-salt loading (Table $2, P<0.01$ ) and $\mathrm{K}$ supplementation lowered them in both SS and SR groups (Table 2, $P<0.01$ ). The levels of

Table 1. Characteristics of salt-sensitive and salt-resistant subjects on baseline

(Mean values and standard deviations)

\begin{tabular}{|c|c|c|c|c|c|}
\hline \multirow[b]{2}{*}{ Parameters } & \multicolumn{2}{|c|}{ SS (21) } & \multicolumn{2}{|c|}{ SR (28) } & \multirow[b]{2}{*}{$P$} \\
\hline & Mean & SD & Mean & SD & \\
\hline Men/women & \multicolumn{2}{|c|}{$9 / 12$} & \multicolumn{2}{|c|}{$14 / 14$} & 0.321 \\
\hline Age (years) & 54.5 & $6 \cdot 3$ & $47 \cdot 6$ & $9 \cdot 2$ & 0.005 \\
\hline Smoking (yes/no) & \multicolumn{2}{|c|}{$7 / 14$} & \multicolumn{2}{|c|}{$11 / 17$} & 0.377 \\
\hline BMI $\left(\mathrm{kg} / \mathrm{m}^{2}\right)$ & $24 \cdot 3$ & $2 \cdot 6$ & $23 \cdot 0$ & 3.1 & 0.137 \\
\hline Waist circumference (cm) & 87.7 & $7 \cdot 3$ & 85.4 & 9.7 & 0.367 \\
\hline Systolic blood pressure (mmHg) & $115 \cdot 2$ & $15 \cdot 3$ & $106 \cdot 8$ & $13 \cdot 0$ & 0.044 \\
\hline Diastolic blood pressure $(\mathrm{mmHg})$ & 74.7 & 8.7 & 71.1 & $8 \cdot 2$ & 0.125 \\
\hline Mean blood pressure $(\mathrm{mmHg})$ & 88.2 & $10 \cdot 2$ & $82 \cdot 8$ & 9.3 & 0.061 \\
\hline Total cholesterol $(\mathrm{mmol} / \mathrm{l})$ & $4 \cdot 3$ & 0.9 & 4.0 & 0.8 & 0.226 \\
\hline TAG $(\mathrm{mmol} / \mathrm{l})$ & 1.4 & 0.8 & 1.2 & 0.5 & 0.342 \\
\hline LDL-cholesterol (mmol/l) & $2 \cdot 4$ & 0.7 & $2 \cdot 2$ & 0.7 & 0.177 \\
\hline HDL-cholesterol (mmol/l) & $1 \cdot 2$ & 0.3 & 1.2 & 0.2 & 0.944 \\
\hline
\end{tabular}

SR, salt resistant; SS, salt-sensitive. 
plasma glucose, insulin and C-peptide in SS subjects were notably higher than that in SR subjects on a high-salt diet (Table 2, $P<0.05$ ).

HOME-IR was considerably elevated from low-salt period to high-salt loading (SS: 1.5 (SD 0.21) v. 2.4 (sD 0.40), $P<0.001$; SR: 1.4 (SD 0.17) v. 1.9 (SD 0.19), $P<0.001$, respectively) and HOME-IR increase induced by a high-salt intake was markedly ameliorated by K supplementation in both SS and SR groups (SS: 2.4 (SD 0.40) v. 1.6 (SD 0.31), $P<0.001$; SR: 1.9 (SD 0.19) v. 1.4 (SD 0.17), $P<0.001$, respectively). In line with plasma glucose, insulin and C-peptide, HOME-IR was higher in SS subjects than that in SR subjects on a high-salt diet (2.4 (sD 0.40) v. 1.9 (sD 0.19), $P<0.05)$.

\section{Potassium supplementation reversed the active effects of salt loading on NLRP3 inflammasome in the subjects}

As shown in Fig. 1, the protein and mRNA levels of NLRP3 $(a, b)$ and Caspase-1 (c, d) in PBMC were significantly increased after high-salt intervention, and $\mathrm{K}$ supplementation reversed them. Moreover, plasma IL- $1 \beta$, the index of NLRP3 inflammasome activation, was also significantly elevated after high-salt loading (e, SS, 8.8 (sD 3.0) v. 16.1 (sD 4.9) pg/ml, $P<0.01$; f, SR, 5.4 (sD 2.7) v. 10.5 (sD 4.0) pg/ml, $P<0.01$ ) and $\mathrm{K}$ supplementation alleviated them (e, SS, 16.1 (sD 4.9) v. 9.8 (sD 3.3) pg/ml, $P<0.01$; f, SR, 10.5 (sD 4.0) v. 5.4 (sD 3.3) pg/ml, $P<0.01$ ). Additionally, plasma IL- $1 \beta$ was considerably higher in SS subjects than that in SR subjects on a high-salt diet (SS, 16.1 (SD 4.9) v. SR, 10.5 (SD 4.0) pg/ml, $P<0.05$ ).

The alteration of insulin resistance index considerably and positively correlated with the change of plasma IL-1 $\beta$ during intervention

As shown in Fig. 2, the net increase in insulin resistance strongly correlated with the net increase in plasma IL- $1 \beta$ after high-salt loading in both SS and SR subjects (a, SS, $r 0 \cdot 748, P<0 \cdot 01$; b, SR, $r 0.575, P=0 \cdot 01$, respectively), and the extent of the reduction in insulin resistance was significantly related to the extent of the decrease in plasma IL- $1 \beta$ from high-salt diet to high-salt diet with K supplementation (c, SS, $r 0.794, P<0.01$; d, SR, $r 0.492$, $P<0 \cdot 01$, respectively).

\section{Potassium supplementation ameliorated salt-loading- induced oxidative stress in the subjects}

Oxidative stress plays a key role in the activation of NLRP3 inflammasome ${ }^{(24-26)}$. To interpret the mechanism by which both salt and $\mathrm{K}$ exert an effect on NLRP3 inflammasome, we further explored the impacts of salt loading and $\mathrm{K}$ supplementation on oxidative stress in the subjects.

As demonstrated in Fig. 3, plasma SOD was significantly decreased after high-salt loading (a, SS, 123.4 (SD 22.1) v. 97.5 (SD $20 \cdot 7) \mu / \mathrm{ml}, P<0.01 ; \mathrm{SR}, 154.6$ (SD 34.4) v. 100.1（SD 23.5) $\mu / \mathrm{ml}$, (a)
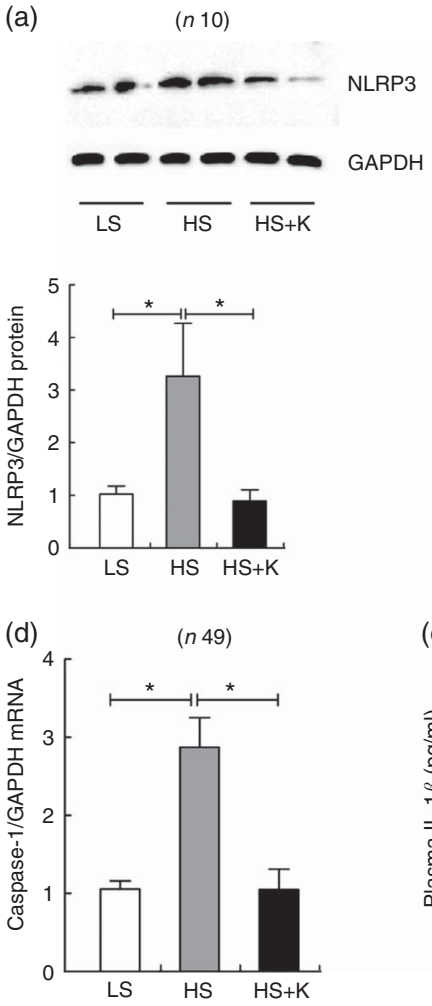

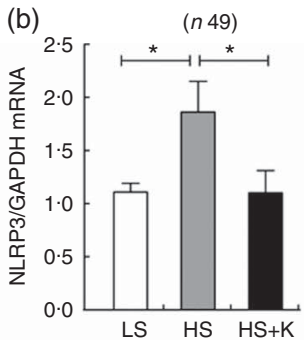

(e)

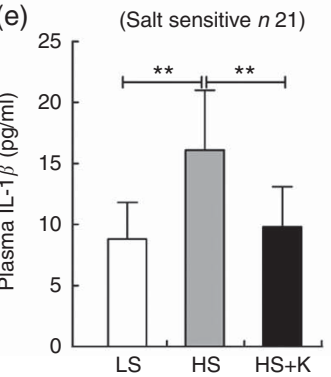

(c)
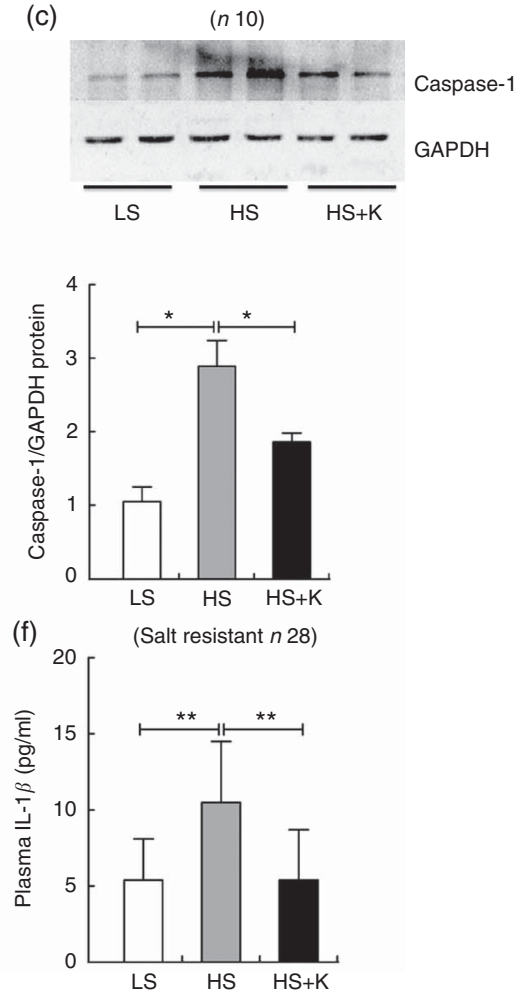

Fig. 1. The impacts of salt loading and potassium supplementation on NLRP3 inflammasome in peripheral blood mononuclear cells (PBMC) from the subjects. The PBMC of the subjects were isolated by Ficoll standard density gradient centrifugation. (a) Representative immunoblot for NLRP3 in PBMC from the subjects. (b) The mRNA level of NLRP3 in PBMC from the subjects by quantitative RT-PCR. (c) Representative immunoblot for caspase-1 in PBMC from the subjects. (d) The mRNA level of caspase-1 in PBMC from the subjects by quantitative RT-PCR. (e) ELISA to detect plasma IL-1 $\beta$ in salt-sensitive subjects. (f) ELISA to detect plasma IL- $1 \beta$ in salt-resistant subjects. Values are means, and standard deviations represented by vertical bars. LS, low salt; HS, high salt; $\mathrm{HS}+\mathrm{K}$, high salt with potassium supplementation. ${ }^{*} P<0.05$, ** $P<0.01$. GAPDH, glyceraldehyde-3-phosphate dehydrogenase. 

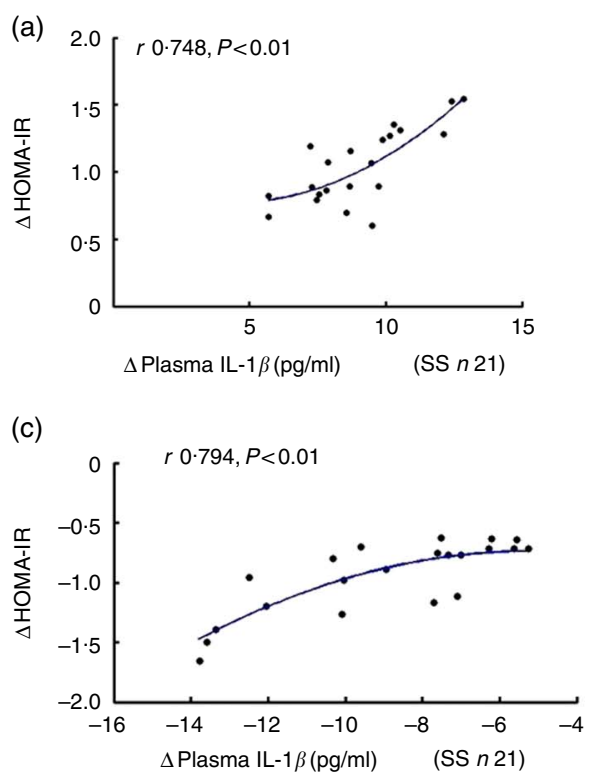
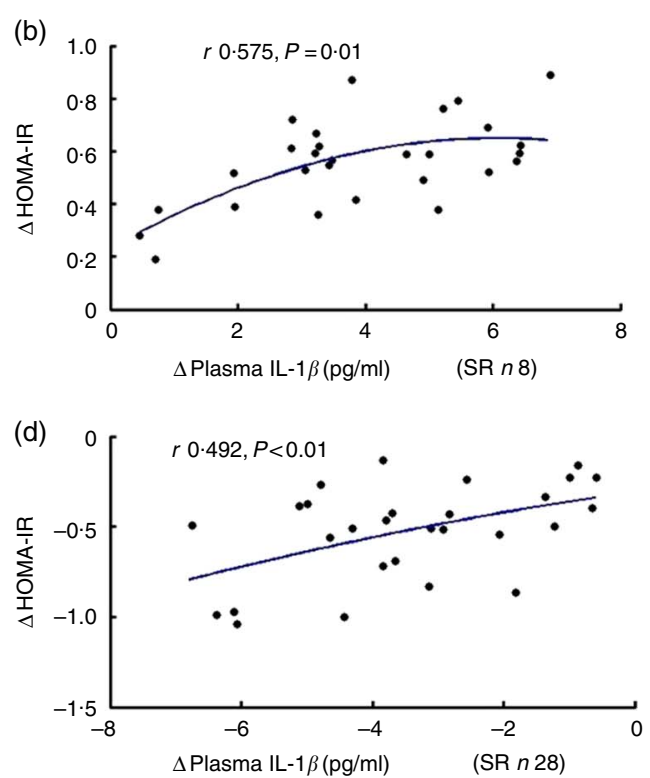

Fig. 2. The correlation between insulin resistance and plasma IL-1 $\beta$. Plasma insulin was detected by radioimmunoassay. Plasma glucose was assayed by the hospital's clinical laboratory. Homeostatic model assessment for insulin resistance index (HOMA-IR) was calculated using HOMA Calculator version 2.2.3 software. The relationship between HOMA-IR and plasma IL-1 $\beta$ after high-salt loading in both salt-sensitive (SS, a) and salt-resistant (SR, b) subjects. The relationship between HOMA-IR and plasma IL-1 $\beta$ in both SS (c) and SR (d) subjects after high-salt loading with potassium supplementation.

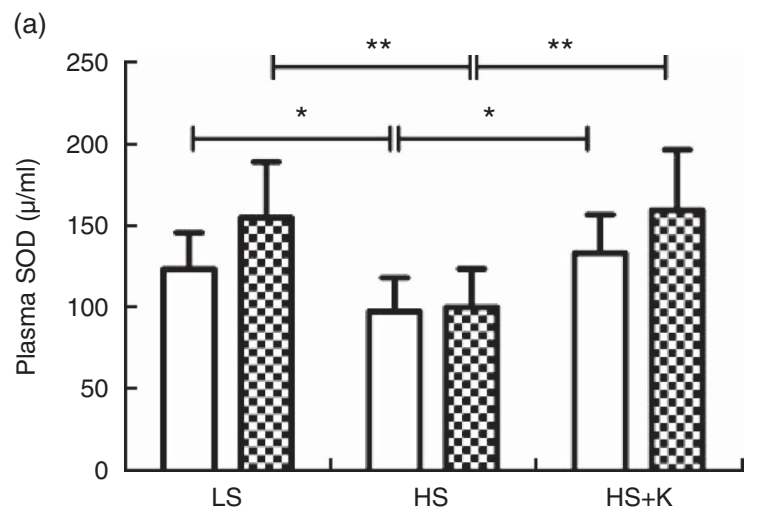

(b)

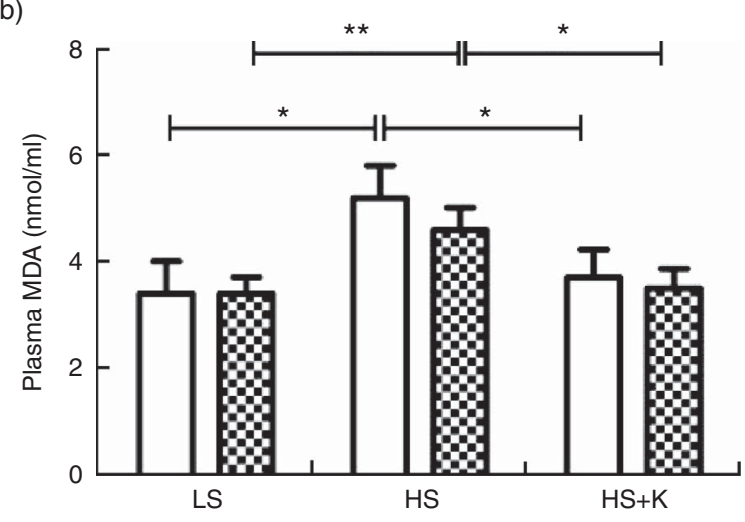

Fig. 3. Potassium supplementation reversed oxidative stress induced by salt loading. Plasma superoxide dismutase (SOD) (a) and plasma malondialdehyde (MDA) (b) were determined by colorimetry. Values are means, and standard deviations represented by vertical bars. $\square$, Salt sensitive; $\mathbb{Q}$, salt resistant; LS, low salt; HS, high salt; $\mathrm{HS}+\mathrm{K}$, high salt with potassium supplementation. ${ }^{*} P<0.05,{ }^{* *} P<0.01$.
$P<0.01$ ) and it was elevated with K supplementation (a, SS, 97.5 (SD 20.7) $v .133 \cdot 1$ (SD 23.7) $\mu / \mathrm{ml}, P<0 \cdot 01 ; \mathrm{SR}, 100 \cdot 1$ (SD 23.5) $v .159 \cdot 2$ (SD $37 \cdot 3) \mu / \mathrm{ml}, P<0 \cdot 01)$. Moreover, high-salt diet elevated plasma MDA (b, SS, 3.4 (sD 0.61) v. $5 \cdot 2(\mathrm{sD} 0.61) \mathrm{nmol} / \mathrm{ml}, P<0 \cdot 01 ; \mathrm{SR}, 3 \cdot 4$ (SD 0.31) v. $4.6(\mathrm{SD} 0.42) \mathrm{nmol} / \mathrm{ml}, P<0.01$ ) and $\mathrm{K}$ supplementation lowered it (b, SS, 5.2 (sD 0.61) v. $3.7(\mathrm{sD} 0.53) \mathrm{nmol} / \mathrm{ml}, P<0.01$; SR, 4.6 (SD 0.42) $v .3 .5(\mathrm{SD} 0.37) \mathrm{nmol} / \mathrm{ml}, P<0.01)$. In addition, plasma SOD in SR subjects was higher than that in SS subjects on a low-salt diet (154.6 (sD 34.4) v. 123.4 (sD 22.1) $\mu / \mathrm{ml}, P<0 \cdot 05$ ). Moreover, plasma MDA in SS subjects was higher than that in SR subjects on a high-salt diet (5.2 (sD 0.61) v. $4.6(\mathrm{SD} 0.42) \mathrm{nmol} / \mathrm{ml}, P<0.05)$.

\section{The change of oxidative stress biomarker significantly levelled} with the alteration of plasma IL-1 $\beta$ during intervention

As shown in Fig. 4, the net increase in plasma IL- $1 \beta$ negatively correlated with the net decrease in plasma SOD after high-salt loading (a, SS, $r-0.661, P=0.001$; b, SR, $r-0.699, P<0.01$, respectively), and the net decrease in plasma IL- $1 \beta$ was negatively related to the net increase in plasma SOD on $\mathrm{K}$ supplementation (c, SS, $r-0.698, P<0.01$; d, SR, $r-0.551, P=0.002$, respectively).

Fig. 5 also displays that the extent of the increase in plasma IL- $1 \beta$ correlated notably with the extent of the increase in plasma MDA after high-salt loading (e, SS, $r$ 0.512, $P=0.018$; f, SR, $r 0.563, P=0.002$, respectively), and the net increase in plasma IL- $1 \beta$ was significantly related to the net increase in plasma MDA on K supplementation (g, SS, $r 0.577, P=0.006$; h, SR, $r 0 \cdot 560, P=0 \cdot 002$, respectively).

\section{Potassium chloride inhibited the active effects of sodium chloride on NLRP3 inflammasome in vitro}

The intervention study demonstrated that salt loading contributed to the activation of NLRP3 inflammasome, and $\mathrm{K}$ 
(a)

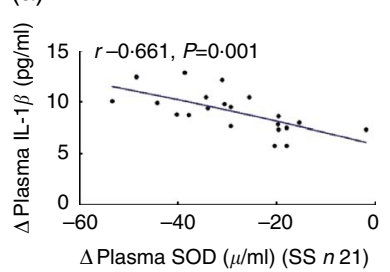

(e)

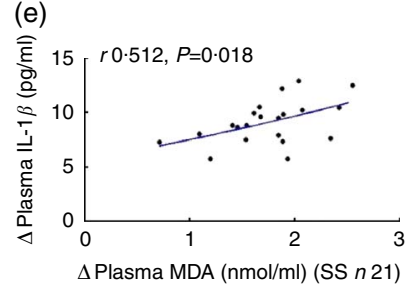

(b)

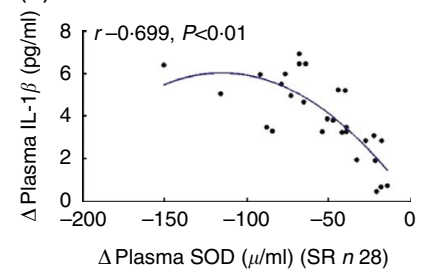

(f)

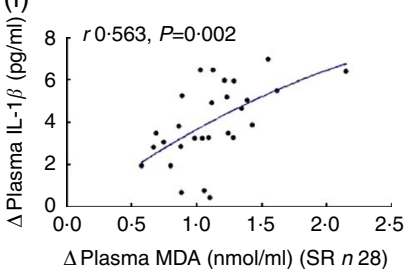

(c)

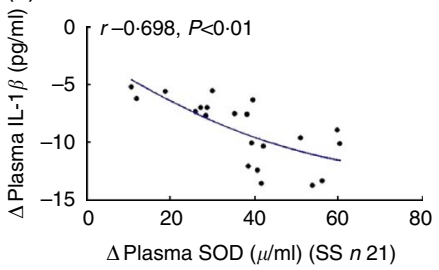

(g)

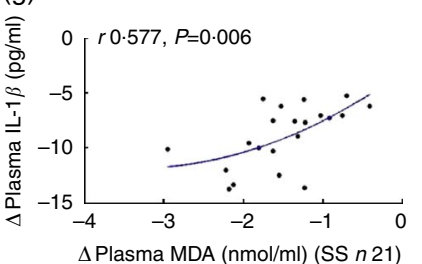

(d)

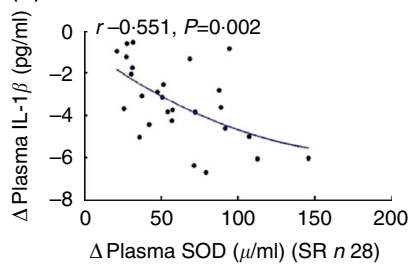

(h)

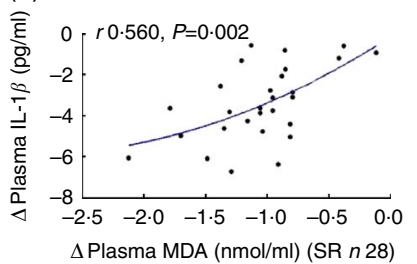

Fig. 4. The correlation between oxidative stress and plasma IL-1 $\beta$. Plasma superoxide dismutase (SOD) and plasma malondialdehyde (MDA) were determined by colorimetry. The plasma IL- $1 \beta$ was assayed by ELISA. The relationship between plasma IL- $1 \beta$ with plasma SOD after high-salt loading (a, salt sensitive (SS); $b$, salt resistant) and high-salt loading with potassium supplementation (c, SS; $d, S R$ ). The relationship between plasma IL-1 $\beta$ with plasma MDA after high-salt loading in both SS (e) and SR (f) subjects, and high-salt loading with potassium supplementation in both SS (g) and SR (h) subjects.

supplementation drastically blocked the activation of NLRP3 inflammasome induced by sodium chloride. To further evaluate the impacts of $\mathrm{Na}$ and $\mathrm{K}$ on NLRP3 inflammasome, we treated the THP-1 cells with different levels of sodium chloride with or without potassium chloride in vitro.

As displayed in Fig. 5, the protein ( $\mathrm{a}$ and b) and mRNA expression ( $c$ and $d$ ) of NLRP3 and the level of culture supernatants IL- $1 \beta$ (e and $\mathrm{f}$ ) was significantly enhanced by sodium chloride in a time- and dose-dependent manner. In line with the results in vivo, potassium chloride considerably prevented the increase in the protein $(\mathrm{g})$ and mRNA expression (h) of NLRP3 and the culture supernatants IL- $1 \beta$ induced by high sodium chloride (i).

\section{Oxidative stress mediated the effects of sodium chloride and potassium chloride on NLRP3 inflammasome in vitro}

The present intervention study showed that salt loading caused the increase in plasma oxidative stress, and $\mathrm{K}$ supplementation radically reversed the effect. Moreover, the change of oxidative stress considerably related with the alteration of plasma IL- $1 \beta$ during intervention. This raises the possibility that oxidative stress is involved in the effects of Na and K on NLRP3 inflammasome. Therefore, to confirm the hypothesis, we further performed the experiments in vitro.

Fig. 6 demonstrated that intracellular ROS was enhanced by sodium chloride in a dose-dependent manner (a) and potassium chloride blocked it (b). Both antioxidant NAC and potassium chloride could significantly reduce the protein (c) and mRNA (d) expression of NLRP3 and the level of culture supernatants IL-1 $\beta$ (e) induced by high sodium chloride. Moreover, potassium chloride could considerably block the increase in the protein (f) and mRNA (g) expression of NLRP3 and the level of culture supernatants IL- $1 \beta(\mathrm{h})$ induced by high sodium chloride or oxidant Rosup. Taken together, these results demonstrated that oxidative stress was involved in the mechanism by which both Na and K exerted an effect on NLRP3 inflammasome.

\section{Discussion}

The present experiments involving normotensive Asians, as well as THP-1 cells, demonstrated that NLRP3 inflammasome was involved in the impacts of salt and $\mathrm{K}$ on insulin resistance. We found that salt loading contributed to intensive oxidative stress, and then induced the activation of NLRP3 inflammasome and consequent production and secretion of IL- $1 \beta$, resulting in insulin resistance, whereas combination of additional $\mathrm{K}$ and salt loading markedly reversed them. On the basis of the crucial role of NLRP3 inflammasome in the induction and progress of insulin resistance ${ }^{(22)}$, we propose that NLRP3 inflammasome has the potential to be a mediator in the impacts of $\mathrm{Na}$ and $\mathrm{K}$ on insulin resistance.

Increasing evidence suggests that compared with prehistoric or primitive humans modern people with more $\mathrm{Na}$ and less $\mathrm{K}$ intake are more vulnerable to suffering from $\mathrm{CVD}^{(2)}$. Although the exact mechanism by which $\mathrm{Na}$ and $\mathrm{K}$ exert the impacts on CVD remains unclear, insulin resistance, a common cornerstone of CVD occurrence and development ${ }^{(36)}$, is recognised as a key player. High Na intake impairs insulin signalling and aggravates insulin resistance in Dahl salt-sensitive rats ${ }^{(10,14)}$, and $\mathrm{K}$ supplementation ameliorates insulin resistance ${ }^{(14)}$. Similarly, highsalt diet enhances insulin resistance in subjects with ${ }^{(11)}$ and without type 2 diabetes ${ }^{(12,13)}$, whereas $\mathrm{K}$ supplementation could improve insulin sensitivity and reduce the prevalence of the metabolic syndrome and type 2 diabetic $^{(15-17,37)}$. Reconciling previous literature with the present report, our result demonstrated that high-salt diet contributed to insulin resistance, whereas adding $\mathrm{K}$ to salt loading markedly reversed the process in the subjects. Thus, the impact of $\mathrm{K}$ supplementation and $\mathrm{Na}$ intake on insulin resistance is just like the yin and yang. It is notable that after high-salt intake, both SS group and SR 
(a)
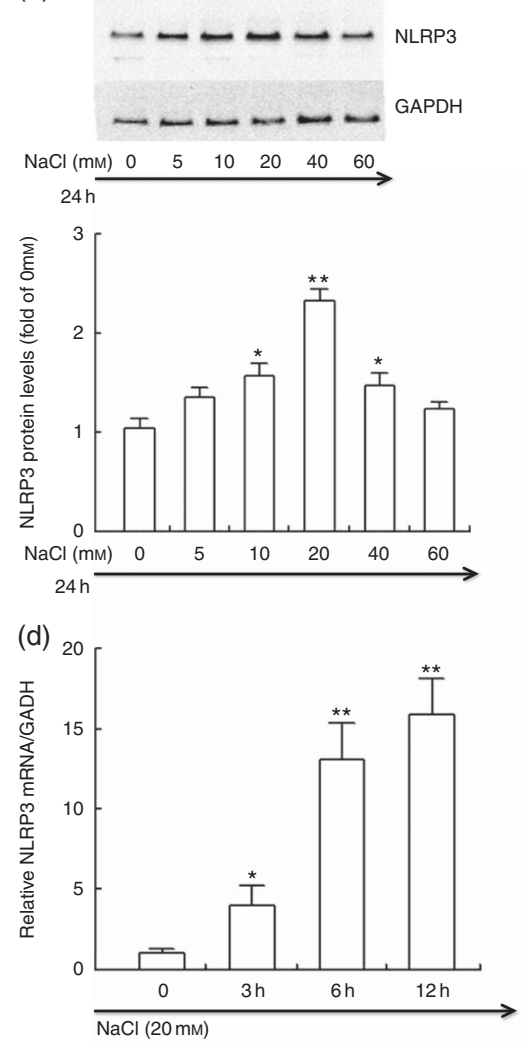

(g)

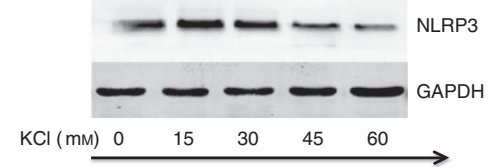

$\mathrm{NaCl}(20 \mathrm{~mm})(24 \mathrm{~h})$

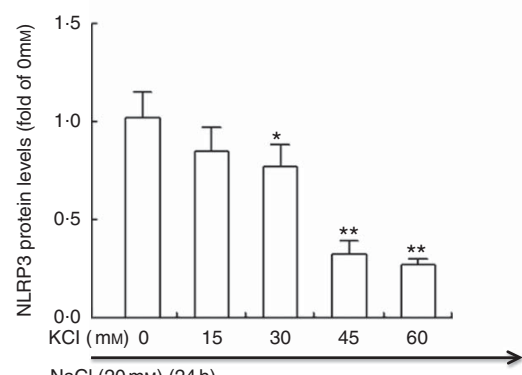

(b)
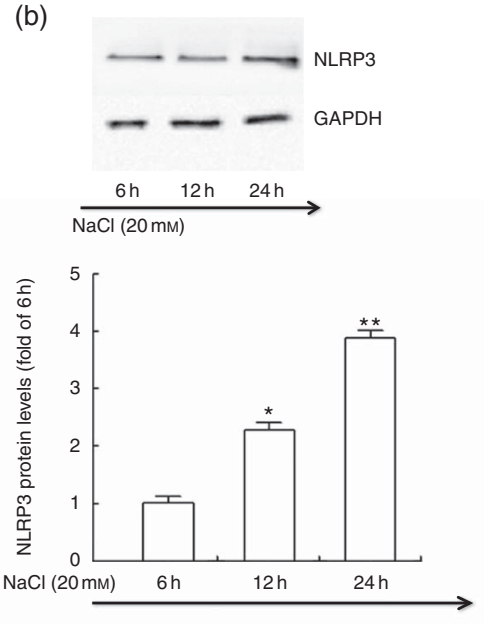

(e)

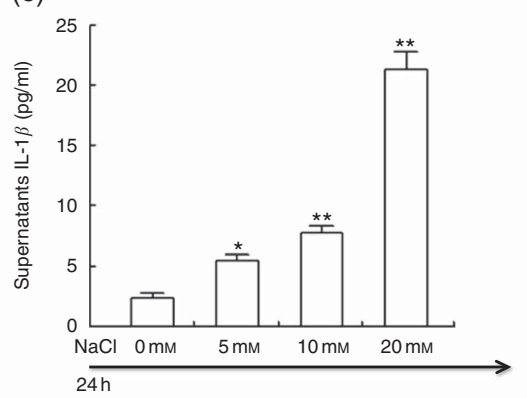

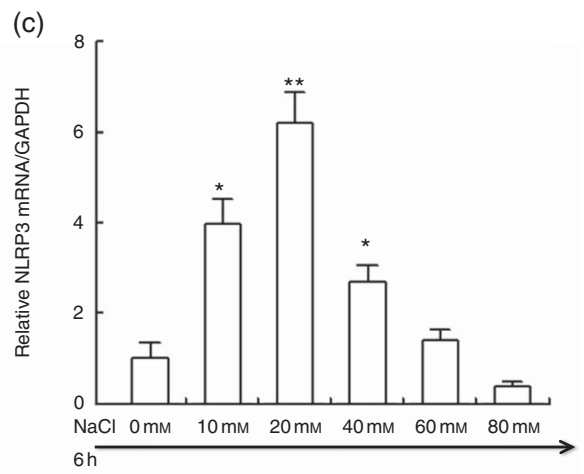

(f)

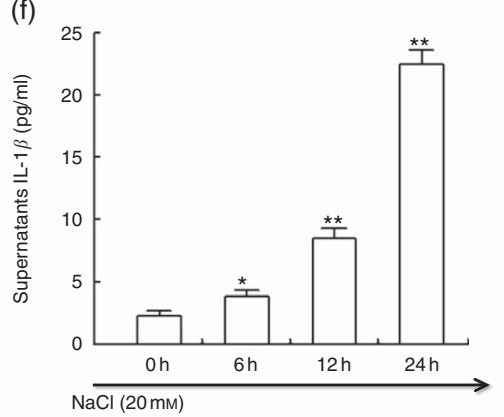

(h)

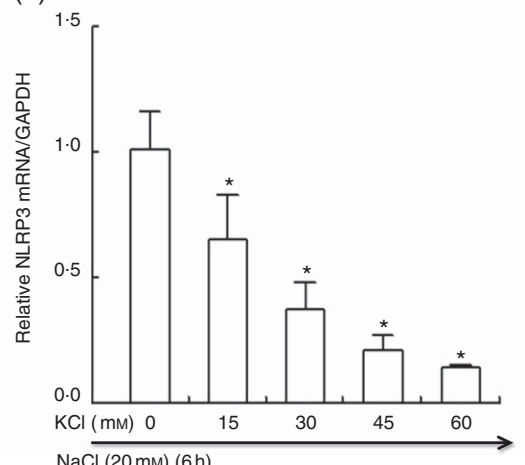

(i)

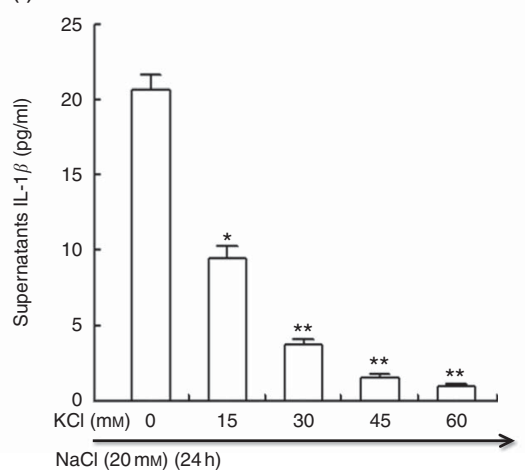

$n 4$ for real-time PCR. $n 3$ for Western blot

Fig. 5. Potassium reversed the active effects of sodium on NLRP3 inflammasome in vitro. THP-1 cells were incubated in Roswell Park Memorial Institute (RPMI) medium containing different levels of sodium chloride with or without potassium chloride. At the end of incubation, cells were lysed, and NLRP3 protein and mRNA expression were determined by Western blotting and real-time PCR, respectively. Culture supernatants were collected and IL-1 $\beta$ was analysed by ELISA. (a, b, g) Representative immunoblot for NLRP3. (c, d, h) The mRNA level of NLRP3 in cultured THP-1 cells by quantitative RT-PCR. (e, $f$, i) ELISA to detect culture supernatants IL-1 $\beta$. Values are means, and standard deviations represented by vertical bars. ${ }^{*} P<0.05$, ${ }^{\star \star} P<0.01$. GAPDH, glyceraldehyde-3-phosphate dehydrogenase.

groups exhibited elevated insulin resistance, whereas some previous experiments revealed that high-salt-induced insulin resistance only in SS individuals or rats. Indeed, our results showed that HOME-IR was higher in SS subjects than in SR subjects on a high-salt diet, indicating that the SS subjects may be more vulnerable to developing insulin resistance following high-salt loading.
With respect to the molecular mechanisms by which both $\mathrm{Na}$ and $\mathrm{K}$ exert an impact on insulin resistance, NLRP3 inflammasome, a large multimeric platform that mediates activation of caspase- 1 and promotes the cleavage of pro-IL- $1 \beta$ into mature forms IL- $1 \beta^{(38,39)}$, may get involved in the process. IL- $1 \beta$ contributes to insulin resistance by impairing insulin signal pathway of adipose, liver and muscle tissues ${ }^{(18-20)}$. Moreover, 
(a)

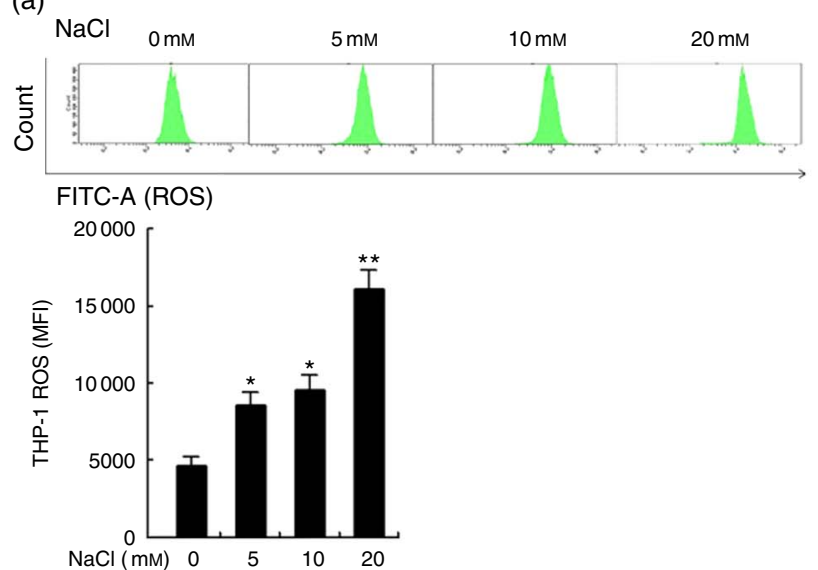

(b)
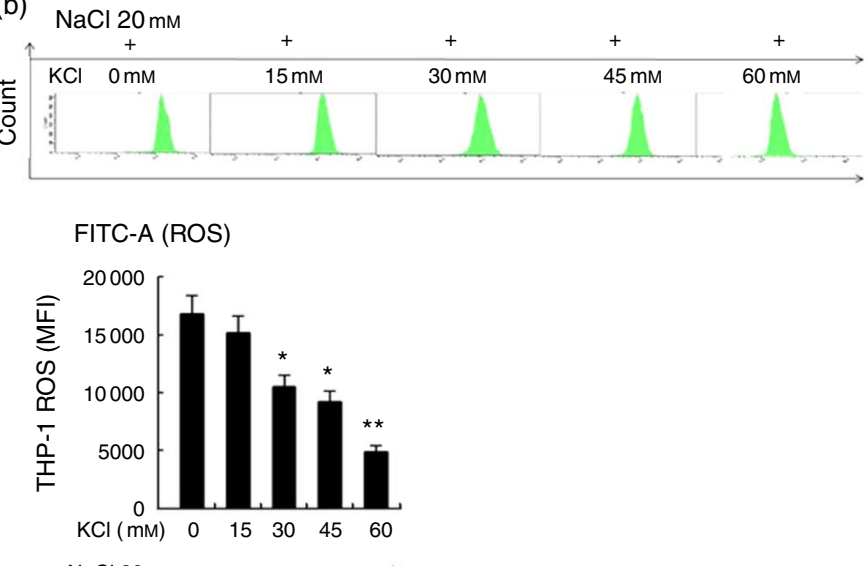

$\mathrm{NaCl} 20 \mathrm{~mm}$ (c)
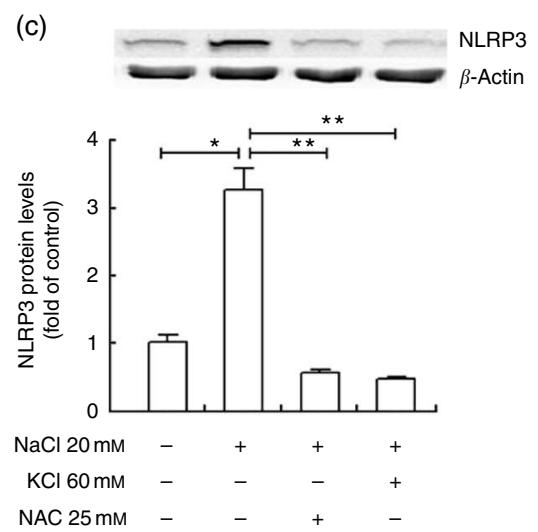

(f)
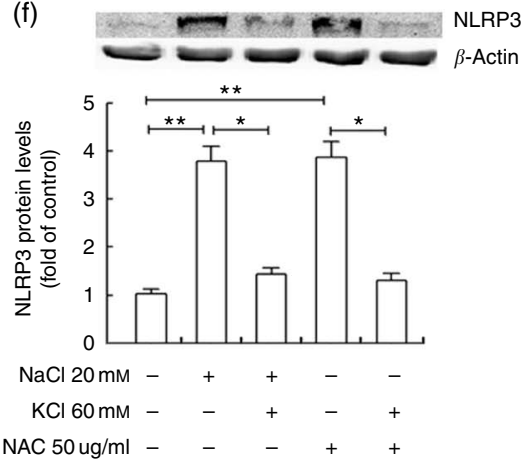

(d)

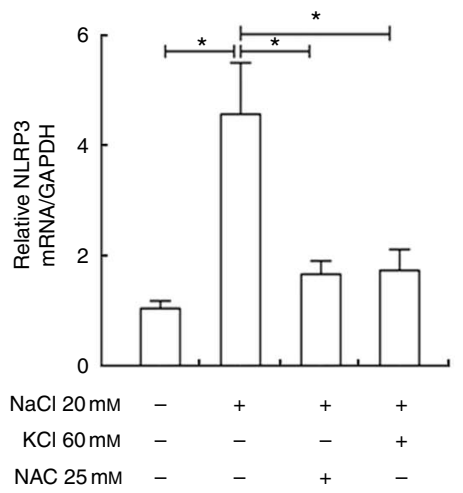

(g)

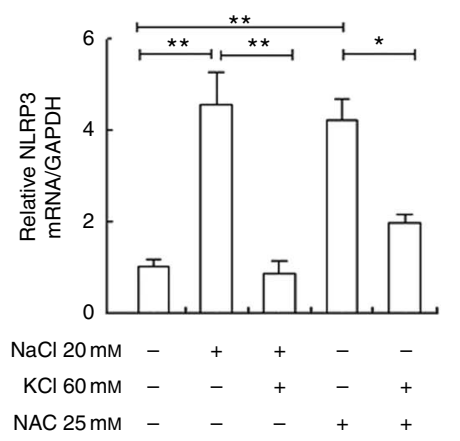

(e)

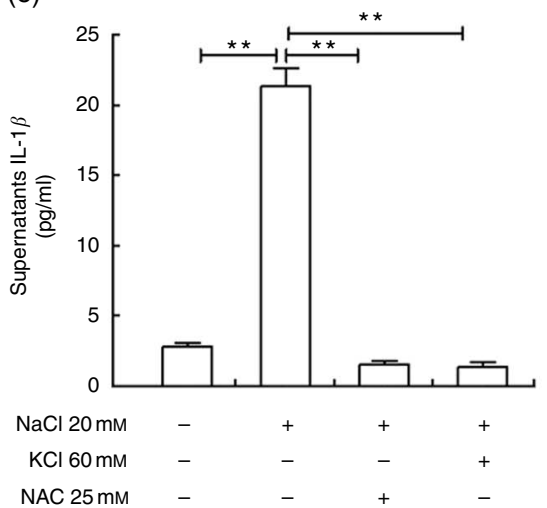

(h)

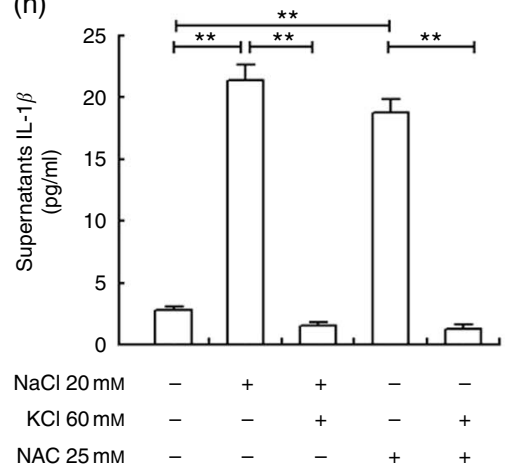

$n 4$ for real-time PCR. $n 3$ for Western blot

Fig. 6. Oxidative stress mediated the effects of sodium and potassium on NLRP3 inflammasome. THP-1 cells were incubated in Roswell Park Memorial Institute (RPMI) with 0-20 mm sodium chloride (6A) or with $20 \mathrm{~mm}$ sodium chloride and 0-60 mm potassium chloride (b) for $6 \mathrm{~h}$. THP-1 cells were incubated in RPMI containing $20 \mathrm{~mm}$ sodium chloride with or without $60 \mathrm{~mm}$ potassium chloride or $25 \mathrm{~mm}$ antioxidant $\mathrm{N}$-acetyl-L-cysteine (NAC) (c-e). Moreover, pre-treated with $60 \mathrm{~mm}$ potassium chloride for $24 \mathrm{~h}$, THP-1 cells were then cultured with oxidant Rosup $(50 \mu \mathrm{g} / \mathrm{ml})$ or additional $20 \mathrm{~mm}$ sodium chloride (f-h). At the end, cells were lysed, and NLRP3 protein and mRNA expression were assayed by Western blotting and real-time PCR, respectively. Culture supernatant IL-1 $\beta$ was analysed by ELISA. (a, b) Representative intracellular reactive oxygen species assayed by flow cytometry. (c, f) Representative immunoblot for NLRP3. (d, g) The mRNA level of NLRP3 in cultured THP-1 cells by quantitative RT-PCR. (e, h) ELISA to detect culture supernatants IL-1 $\beta$. Values are means, and standard deviations represented by vertical bars. ${ }^{*} P<0.05,{ }^{* *} P<0.01$. GAPDH, glyceraldehyde-3-phosphate dehydrogenase.

previous studies in mice with gene ablation have indicated that NLRP3 inflammasome is a central player in the initiation and development of insulin resistance $e^{(18,20,22,23,25)}$. Genetic knockout of NLRP3 or its associated molecules relieves insulin resistance induced by a high-fat diet. Accordingly, the present study focused on assessing the effects of salt and K on NLRP3 inflammasome in vivo and in vitro. As expected, high salt significantly contributed to the activation of NLRP3 inflammasome and additional $\mathrm{K}$ markedly reversed the effect in both the subjects and THP-1 cells. Moreover, insulin resistance notably related with the activity of NLRP3 inflammasome in the subjects. Taken together, these results suggest that $\mathrm{Na}$ and $\mathrm{K}$ 
exert completely inverse effects on NLRP3 inflammasome activity.

Notably, high-salt diet causes increased oxidative stress $^{(27-30)}$, whereas diets abundant in $\mathrm{K}$ lessens oxidative stress induced by salt loading ${ }^{(31)}$. Moreover, $\mathrm{K}$ can inhibit ROS generation of human white blood cells in vitro ${ }^{(32)}$. In line with previous reports, our results demonstrated that high salt significantly increased oxidative stress and additional $\mathrm{K}$ considerably reduced oxidative stress in both the subjects and THP-1 cells. Multiple signalling pathways of NLRP3 inflammasome activation converges on ROS production. NLRP3 inflammasome is a common sensor of cellular stress through its activation by ROS. Either various danger signals or environmental irritants activate NLRP3 inflammasome through promoting ROS production $^{(24-26)}$. NAC, an ROS inhibitor, significantly prevents NLRP3 inflammasome activation and IL- $1 \beta$ secretion induced by LPS plus palmitate-free bovine serum albumin ${ }^{(18)}$. Similarly, the present experiment showed that either additional $\mathrm{K}$ or NAC significantly inhibited NLRP3 inflammasome activation induced by high salt or oxidant Rosup in vitro. Taken together, these results demonstrate that oxidative stress is involved in the modulation of NLRP3 inflammasome activity by $\mathrm{Na}$ and $\mathrm{K}$.

Furthermore, recent report demonstrates that $\mathrm{K}$ efflux and $\mathrm{Na}$ influx can modulate NLRP3 inflammasome activation ${ }^{(40)}$, and ROS production is often accompanied by $\mathrm{K}_{\text {efflux }}{ }^{(24)}$. It is possible that ROS generation triggers intracellular $\mathrm{K}$ efflux or vice versa $^{(41)}$. Therefore, we cannot exclude the possibility that high salt activates NLRP3 inflammasome in part by promoting $\mathrm{Na}$ influx and/or $\mathrm{K}$ efflux and additional $\mathrm{K}$ inhibits NLRP3 inflammasome to some extent by blocking $\mathrm{K}$ efflux.

\section{Conclusions}

In summary, the present study demonstrated that high salt contributed to insulin resistance by oxidative stress activation of NLRP3 inflammasome, whereas additional K released high-saltinduced insulin resistance via the inhibition of oxidative stress and NLRP3 inflammasome. Thus, we propose that NLRP3 inflammasome may be a common mediator by which $\mathrm{Na}$ and $\mathrm{K}$ exert an impact on insulin resistance. In addition, $\mathrm{K}$ supplementation appears to help to improve insulin resistance via inhibiting NLRP3 inflammasome and reduce the occurrence and development of CVD in modern people with high-salt diets. $\mathrm{K}$ is abundant in fresh fruits and vegetables. Therefore, a greater fresh fruit and vegetable consumption and reasonable salt restriction could protect against the occurrence of insulin resistance and CVD.

\section{Acknowledgements}

This work was supported in part by the National Program on Key Basic Research Project of China (973 Program: 2012 CB517804 to Z. Y.); the National Science Fund for Distinguished Young Scholars (nos 81025002 to Z. Y.); and NSFC (nos 81370357 and 81570381 to J. M.).

The authors' contributions are as follows: Z. W. wrote the manuscript; Z. W., W. W., K. R., D. Z., J. L., Y. W. and J. Z. participated in the study design and performed all processes of the study; Z. Y. and J. M. provided supervision and revised the manuscript; D. Z., J. L., Y. W. and J. Z. assisted with the editing of the manuscript; Z. W., W. W. and K. R. were involved in data acquisition, recruited subjects and collected samples. All authors participated in the analytic discussion of the results and approved the final version of the manuscript.

None of the authors has any conflicts of interest to declare.

\section{References}

1. Gardener H, Rundek T, Wright CB, et al. (2012) Dietary sodium and risk of stroke in the Northern Manhattan study. Stroke 43, 1200-1205.

2. Cook NR, Cutler JA, Obarzanek E, et al. (2007) Long term effects of dietary sodium reduction on cardiovascular disease outcomes: observational follow-up of the trials of hypertension prevention (TOHP). BMJ 334, 885-888.

3. Bibbins-Domingo K, Chertow GM, Coxson PG, et al. (2010) Projected effect of dietary salt reductions on future cardiovascular disease. $N$ Engl J Med 362, 590-599.

4. He FJ \& MacGregor GA (2011) Salt reduction lowers cardiovascular risk: meta-analysis of outcome trials. Lancet $\mathbf{3 7 8}$, 380-382.

5. D'Elia L, Barba G, Cappuccio FP, et al. (2011) Potassium intake, stroke, and cardiovascular disease a meta-analysis of prospective studies. J Am Coll Cardiol 57, 1210-1219.

6. Aburto NJ, Hanson S, Gutierrez H, et al. (2013) Effect of increased potassium intake on cardiovascular risk factors and disease: systematic review and meta-analyses. BMJ $\mathbf{3 4 6}$, f1378.

7. Fang Y, Mu JJ, He LC, et al. (2006) Salt loading on plasma asymmetrical dimethylarginine and the protective role of potassium supplement in normotensive salt-sensitive Asians. Hypertension 48, 724-729.

8. Ginsberg HN (2000) Insulin resistance and cardiovascular disease. J Clin Invest 106, 453-458.

9. Bonora E, Kiechl S, Willeit J, et al. (2007) Insulin resistance as estimated by homeostasis model assessment predicts incident symptomatic cardiovascular disease in Caucasian subjects from the general population: the Bruneck study. Diabetes Care 30, 318-324.

10. Ogihara T, Asano T, Ando K, et al. (2001) Insulin resistance with enhanced insulin signaling in high-salt diet-fed rats. Diabetes 50, 573-583.

11. Vedovato M, Lepore G, Coracina A, et al. (2004) Effect of sodium intake on blood pressure and albuminuria in type 2 diabetic patients: the role of insulin resistance. Diabetologia 47, 300-303

12. Millen AM, Norton GR, Majane $\mathrm{OH}$, et al. (2013) Insulin resistance and the relationship between urinary $\mathrm{Na}(+) / \mathrm{K}(+)$ and ambulatory blood pressure in a community of African ancestry. Am J Hyperten 26, 708-716.

13. Baudrand R, Campino C, Carvajal CA, et al. (2014) High sodium intake is associated with increased glucocorticoid production, insulin resistance and metabolic syndrome. Clin Endocrinol 80, 677-684.

14. Ogihara T, Asano T, Ando K, et al. (2002) High-salt diet enhances insulin signaling and induces insulin resistance in Dahl salt-sensitive rats. Hypertension 40, 83-89.

15. Teramoto T, Kawamori R, Miyazaki S, et al. (2011) Sodium intake in men and potassium intake in women determine the prevalence of metabolic syndrome in Japanese hypertensive patients: OMEGA Study. Hypertens Res 34, 957-962.

16. Lee H, Lee J, Hwang SS, et al. (2013) Potassium intake and the prevalence of metabolic syndrome: the Korean National 
Health and Nutrition Examination Survey 2008-2010. PLOS ONE 8, e55106.

17. Chatterjee R, Colangelo LA, Yeh HC, et al. (2012) Potassium intake and risk of incident type 2 diabetes mellitus: the Coronary Artery Risk Development in Young Adults (CARDIA) Study. Diabetologia 55, 1295-1303.

18. Wen H, Gris D, Lei Y, et al. (2011) Fatty acid-induced NLRP3ASC inflammasome activation interferes with insulin signaling. Nat Immunol 12, 408-415.

19. Wen H, Ting JP \& O'Neill LA (2012) A role for the NLRP3 inflammasome in metabolic diseases - did Warburg miss inflammation? Nat Immunol 13, 352-357.

20. Stienstra R, Joosten LA, Koenen T, et al. (2010) The inflammasome-mediated caspase- 1 activation controls adipocyte differentiation and insulin sensitivity. Cell Metab 12, 593-605.

21. Mandrup-Poulsen T, Pickersgill L \& Donath MY (2010) Blockade of interleukin 1 in type 1 diabetes mellitus. Nat Rev Endocrinol 6, 158-166.

22. Stienstra R, van Diepen JA, Tack CJ, et al. (2011) Inflammasome is a central player in the induction of obesity and insulin resistance. Proc Natl Acad Sci U S A 108, 15324-15329.

23. Vandanmagsar B, Youm YH, Ravussin A, et al. (2011) The NLRP3 inflammasome instigates obesity-induced inflammation and insulin resistance. Nat Med 17, 179-188.

24. Tschopp J \& Schroder K (2010) NLRP3 inflammasome activation: the convergence of multiple signalling pathways on ROS production? Nat Rev Immunol 10, 210-215.

25. Zhou R, Tardivel A, Thorens B, et al. (2010) Thioredoxininteracting protein links oxidative stress to inflammasome activation. Nat Immunol 11, 136-140.

26. Dostert C, Petrilli V, Van Bruggen R, et al. (2008) Innate immune activation through Nalp3 inflammasome sensing of asbestos and silica. Science 320, 674-677.

27. Di Castro S, Scarpino S, Marchitti S, et al. (2013) Differential modulation of uncoupling protein 2 in kidneys of strokeprone spontaneously hypertensive rats under high-salt/lowpotassium diet. Hypertension 61, 534-541.

28. Meng S, Cason GW, Gannon AW, et al. (2003) Oxidative stress in Dahl salt-sensitive hypertension. Hypertension $\mathbf{4 1}$, $1346-1352$.
29. Meng S, Roberts LJ 2nd, Cason GW, et al. (2002) Superoxide dismutase and oxidative stress in Dahl salt-sensitive and -resistant rats. Am J Physiol Regul Integr Comp Physiol 283, R732-R738.

30. Wang Y, Chen AF \& Wang DH (2006) Enhanced oxidative stress in kidneys of salt-sensitive hypertension: role of sensory nerves. Am J Physiol Heart Circ Physiol 291, H3136$\mathrm{H} 3143$.

31. Ishimitsu T, Tobian L, Sugimoto K, et al. (1996) High potassium diets reduce vascular and plasma lipid peroxides in stroke-prone spontaneously hypertensive rats. Clin Exp Hypertens 18, 659-673.

32. McCabe RD, Bakarich MA, Srivastava K, et al. (1994) Potassium inhibits free radical formation. Hypertension 24, 77-82.

33. Chen J, Gu D, Huang J, et al. (2009) Metabolic syndrome and salt sensitivity of blood pressure in non-diabetic people in China: a dietary intervention study. Lancet 373, 829-835.

34. Gu D, Zhao Q, Chen J, et al. (2013) Reproducibility of blood pressure responses to dietary sodium and potassium interventions: the GenSalt study. Hypertension 62, 499-505.

35. Sullivan JM (1991) Salt sensitivity. Definition, conception, methodology, and long-term issues. Hypertension 17, I61-I68.

36. Saltiel AR \& Olefsky JM (2017) Inflammatory mechanisms linking obesity and metabolic disease. J Clin Invest 127, 1-4.

37. Shin D, Joh HK, Kim KH, et al. (2013) Benefits of potassium intake on metabolic syndrome: The fourth Korean National Health and Nutrition Examination Survey (KNHANES IV). Atherosclerosis 230, 80-85.

38. Schroder K \& Tschopp J (2010) The inflammasomes. Cell 140, 821-832.

39. Barker BR, Taxman DJ \& Ting JP (2011) Cross-regulation between the IL-1beta/IL-18 processing inflammasome and other inflammatory cytokines. Curr Opin Immunol 23, 591-597.

40. Munoz-Planillo R, Kuffa P, Martinez-Colon G, et al. (2013) K (+) efflux is the common trigger of NLRP3 inflammasome activation by bacterial toxins and particulate matter. Immunity 38, 1142-1153.

41. Kowaltowski AJ, de Souza-Pinto NC, Castilho RF, et al. (2009) Mitochondria and reactive oxygen species. Free Radic Biol Med 47, 333-343. 